\title{
Medical Education in the 21st Century: Students Driving the Global Agenda
}

\author{
S. Singh, J. McCool, J. Weller, and A. Woodward \\ Faculty of Medical and Health Sciences, University of Auckland, Auckland 1142, New Zealand \\ Correspondence should be addressed to S. Singh, sudhvir@gmail.com
}

Received 15 December 2011; Revised 5 March 2012; Accepted 6 March 2012

Academic Editor: Susan Hallam

Copyright $\odot 2012$ S. Singh et al. This is an open access article distributed under the Creative Commons Attribution License, which permits unrestricted use, distribution, and reproduction in any medium, provided the original work is properly cited.

This descriptive article examines the potential for student-led initiatives in international health to be better integrated with formal medical education systems. Students have embraced the challenges and opportunities provided by globalisation to take a leadership role on international issues. Medical students are involved with a diverse portfolio of international activities, including work to internationalise the medical curriculum, the establishment of "hands-on" development projects, efforts to promote student exchanges, and engagement with high-level international policy fora. Such experiences not only add to the personal and professional development of the individual student, but also have the potential to contribute to the academic environment of the host institution as well as more broadly influencing the determinants of international health outcomes. There are challenges and risks associated with independent student initiatives, however these risks can be mitigated if institutions work in partnership with their students and peers internationally.

Health is no longer a parochial affair. Many of the problems are plainly international-importation of infectious diseases, and the spread of noncommunicable diseases by the global marketing of tobacco, alcohol, and nutrient poor foods are obvious examples. But there are solutions that can operate on the same scale. In the past medical students were educated principally in the setting and context of their local community. Now they have the capacity to respond to international health challenges through advances in information sharing and connectivity that were unimagined a generation ago. Through their networks and the opportunities for international engagement that arise in the medical course, students can now take a leadership role on global issues. It is important to acknowledge the risks: little is known about the efficacy and ethical suitability of existing student-led initiatives. This descriptive paper examines ways in which students can, with appropriate institutional support, contribute to medical education that is fit for purpose in the 21st century.

In the past, most universities expected their graduates to practise close to where they were trained, so students were taught what was thought necessary to serve their local communities. There were exceptions: many European universities, for instance, have placed a strong emphasis on international experience. But frequently students had little exposure to medicine outside their own geographical environment, and information flow between nations was limited. Teaching reflected the prevailing culture of the local region. As a result of globalization, we have moved to an era in which students are far more mobile, can effortlessly tap into information from anywhere in the world, and create and maintain networks with peers in almost every country. Medical schools also put more effort now into recruiting international students, for a variety of reasons (including fees), and these students can expand their local colleagues' horizons.

The health impacts of climate change, the potential of global trade mechanisms for tobacco control, and the speed of infectious disease pandemics are just some of the reminders of how the determinants of health are much expanded. The modern doctor must have an understanding of the languages, cultures, and practical arrangements of a world beyond the geographical boundaries of her or his local region [1]. Incorporating international health themes 
in medical education is not merely an altruistic pursuit, but offers tangible benefits to institutions through encouraging research collaboration, improving the public image of the host institution, and reducing the risk of communicable disease threats at a regional level [2]. For vocational reasons, the 21 st century medical education systems need to train doctors fit to work in an international context, and ready to respond to global health issues [3]. But medical education may benefit in other respects from an internationalized curriculum. Exposure to the great variety of ways in which medicine in practised, and the different environments in which doctors work, should encourage students to be more thoughtful about their own circumstances and more critical of local received wisdom.

The amount of material that could be included in the medical curriculum grows, year by year, at an exponential rate. One response to the crowding problem is a push for developing critical appraisal skills, now seen to be as important as the retention of medical facts. For example, academics at the University of Emory have introduced problem-based "case competitions" to teach global health, moving beyond instructor-led didactic sessions [4]. Another step has been the move to incorporate broader evaluative and judgementassisting disciplines. This is well illustrated by the United Kingdom (UK) General Medical Council's report Tomorrow's Doctors, which called for increasing educational priority to be given to "the wide range of cultural, environmental and ethical issues that will increasingly impinge on the problems of health" [5]. Similarly, the Accreditation Council for Graduate Medical Education (ACGME) in the USA has transitioned graduate medical education (GME) assessment away from traditional standardised testing, clinical experience records, and evaluations of clinical skills, to a competency assessment model addressing defined standards of performance [6]. We argue that this transformation needs to continue with the incorporation of broader themes relevant to globalization and international health. This process of globalizing the curriculum is consistent with the "reconceptualisation movement" of curriculum development, whereby curricula are treated as processes rather than content [7]. This movement has been largely neglected by institutions that train health professionals [8] and is in line with the work of the World Federation for Medical Education (WFME) in developing an international consensus on global standards for basic medical education [9]. One such standard specifies that institutions should pursue international exchanges of academic staff and students.

Currently, many international medical students in the developed world have an opportunity to travel abroad for the "elective" component of their training, which is frequently the only exposure a graduate has to front line international health. Otherwise, there are few mechanisms for preparing graduates to respond to the challenges confronted when experiencing medicine in a global context [1]. Although students enter the medical programme more cognisant of international affairs through exposure to global media networks, personal travels and interactions with an increasingly international student cohort, these experiences alone cannot compete with the reality of dealing with health issues in resource poor settings, or anticipating the reach of "international" problems in a local context. Some have argued that, in isolation, international medical electives do little to address the underlying causes of global inequalities and are far more beneficial for the student than the recipient community [10]. However, there is evidence that returning elective students is beneficial for their local communities, with greater proportions of internationally exposed students choosing to train in hard-to-staff disciplines such as primary care and practising amongst disadvantaged communities compared to their solely locally trained colleagues [11]. Thus, there is potential for educators to better integrate the elective experience into the training pathway to improve outcomes for both students and host institutions. There is also scope for further collaboration between elective programmes and both nongovernmental and governmental institutions to ensure that the overseas elective is implemented in a productive manner that contributes towards positive health and education outcomes.

As a response to the growing understanding of the international dimensions of medicine and the belief that the medical profession has a role in health advocacy beyond the clinic, students have initiated their own activities to engage with international health, outside of formal curricula. Student groups have played a part in initiating community engagement and supporting international health projects [12]. Several organisations have been established that encourage student engagement with international affairs during not just their elective term but throughout their training $[13,14]$. These initiatives have the capacity to effect positive change and promote an international approach to health. When engaging with these activities, students develop skills in advocacy, communication, aid provision, political campaigning, audit, and the ability to work with multidisciplinary teams. Such groups allow students to not only demonstrate leadership but also to learn fundamental skills relevant to medical training, often from senior doctors and allied health professionals who are actively involved with international fora. For example, the Nobel Peace Prize laureate International Physicians for the Prevention of Nuclear War includes a dynamic global student movement, which allows medical students to learn from senior doctors by collaborating on a wide range of projects and activities, including academic writing and political engagement. Despite occurring outside the framework of formal medical education, these activities are nonetheless relevant to the professional development of the medical workforce.

The Fiji Village Project (FVP) is an example of an international student-led humanitarian project. The project was begun in 2007 by medical students from Australia, New Zealand, and Fiji, with the aim of using a collaborative approach to address basic public health deficiencies, such as lack of safe water supplies, in a village setting, [15]. The World Health Organization (WHO) and the local academic department of Public Health at the Fiji National University have endorsed the project and provide academic and logistical support. Key activities in the buildup to each project include engaging with the village community, performing public health assessments to determine 
the appropriate interventions, evaluating previous projects, and fundraising and awareness raising activities in the home countries. This preparation culminates in a "hands on" programme where students work with the village to establish and maintain new water supplies, screen villagers for both communicable and noncommunicable diseases, build public health-related capacity within the village, and administer a school education programme. There is a need for formal assessment of the outcomes and efficacy of student-led initiatives such as the FVP however anecdotal experience suggests that these projects have the potential to deliver immense personal, clinical and educational benefits for students as well as potentially improving the health and quality of life of the local community.

There are numerous other examples of medical studentled projects, many of which come under the umbrella of the International Federation of Medical Students' Associations (IFMSA). The IFMSA is one of the largest nongovernmental organisations in the world, with over 1.2 million members from over 100 nations, represented through their national medical student associations. The WHO recognises the IFMSA as the primary representative body for international medical students, which allows the IFMSA to have influence in promoting global health through a variety of UN-related bodies, as well as organising independent projects and exchanges. For example, the IFMSA has led projects to raise awareness of neglected tropical diseases in Latin America [16], migrants' right to health [17], and the relationship between nutrition and health in Japan [18].

In addition to developing international projects and networks, student groups have acted to consolidate their role as stakeholders in curriculum development and pushed for greater acknowledgment of themes related to globalization and international health in their formal learning. The need for proactive engagement between institutions and student organisations has been acknowledged in the WFME standards for basic medical education [9]. For example, medical students in Canada generated a framework for the incorporation of peace and health in the curriculum [19]. In response to burgeoning epidemics in both obesity and malnutrition globally, American medical students identified a gap in their medical education and took it upon themselves to develop national guidelines for nutrition in medical curricula [20]. Building on the work of bodies like the UK General Medical Council, the IFMSA developed core curriculum standards in 2006 [21]. This framework underlined "the importance of including environmental, cultural and international healthrelated issues in our medical curriculum" (Page 272). Medical students have also acted to promote the democratization of access to medical information around the world [22].

The issue of climate change provides a salient example of the potential for medical student movements to have influence at a global level. Many medical students are passionate about engaging in climate change advocacy, as it is an issue that is truly global in nature, which has the potential to exacerbate existing international health threats. The long half-life of carbon emissions and the timeframes associated with predicted climate change-related impacts draw on principles of intergenerational justice which are particularly relevant to the medical student demographic. Furthermore, the relationship between climate change and human health has received relatively little attention in mainstream political discourse, thus medical students sense an opportunity to influence the climate change narrative and develop novel, impactful solutions [23]. Medical students have developed a variety of national campaigns as well as engaging with the United Nations Framework Convention on Climate Change (UNFCCC). Each year there are medical student delegations at the Conference of the Parties (COPs), the peak decision making body of the UNFCCC, and medical student groups also make submissions on relevant agenda items throughout the year. This engagement has been recognised by both the WHO and various organs within the UNFCCC, who consider medical students to be a major contributor to climate change related discourse:

\begin{abstract}
"Comprehensive awareness-raising involves a diverse multiplicity of actors... These actors include major societal groups such as youth, women, NGOs. . . medical students and engineers" [24].
\end{abstract}

Student-led international initiatives pose several challenges, many of which could be overcome if these activities were better aligned with formal medical education systems. As with elective programmes, student-led projects in foreign communities can inadvertently trigger ethical hazards [25, 26]. These projects may be one off in nature, utilise nonevidenced based methodology, and can be implemented without adequate consultation with the local community. Students must avoid imposing paternalistic values and "Western" models of care when approaching development work. Indeed, some authors link global medical education patterns to the process of neocolonialism [27]. International best practice guidelines for global health training outline several standards that should be met by students and institutions engaging in international health initiatives [26]. Student groups working in isolation are in a difficult position to meet these guidelines, particularly standards specifying effective mentorship and supervision.

Given the turnover of students as they progress through training, the sustainability of initiatives becomes a challenge, and without adequate succession planning, institutional knowledge can easily be lost and the essential step of programme evaluation can be neglected. Innovation can peak during the establishment phase of a new initiative, and maintenance of the initial levels of creativity and passion can be challenging as projects expand and are handed over to new cohorts of students.

Resourcing is a universal constraint, often curtailing the ability of projects to live up to their potential. With the frequent emergence of new initiatives, the governance and integration of student-led initiatives becomes a challenge. When activities are duplicated without adequate oversight, the efficiency of organisation and outreach can decline as groups compete for personnel and funding. On the other hand, overly robust oversight of student activities by conglomerate associations can limit the development of grassroots initiatives as well as the incorporation of new ideas and 
personnel to student movements. A further challenge lies in the potential for international programmes to contribute to the "brain drain" of health professionals from less developed countries to more developed countries.

These core challenges can be alleviated if formal medical education systems embrace student-led initiatives. Institutions of medical training derive tremendous indirect benefits from the independent efforts of their students and therefore institutions have the incentive to engage with student activities. When students travel abroad or speak within their own communities through a student organisation, they act as de facto ambassadors for their institution, providing a great forum for the promotion of the institution's brand, which is particularly important in the age of increasing tuition fees and competitive international ranking systems for universities. If leading academics-mentored student leaders and projects were closely aligned with their host institutions, there is the potential for greater academic citations and more opportunities for alumni involvement and fundraising. Moreover, student organisations frequently attract high profile speakers to their host institutions, which again promote both the brand and academic environment of the institution.

We therefore call for medical institutions to embrace student-led initiatives that promote international understanding and advocacy and to better integrate these activities into curricula. This needs to be in addition to fostering broader programmes in global health teaching and research and providing logistical support for students contemplating an international elective term. Research-led medical institutions have experience addressing ethical concerns and ensuring the sustainability of long term research programmes and this expertise can be used to provide formal guidance in ethics and planning for international student projects [25].

To this end, international best-practice guidelines for global health experience can be implemented by institutions to ensure student projects operate in a manner that accounts for ethical hazards [26]. Whilst preserving the grassroots and independent nature of student movements, formal medical education systems can also play a key role in quality control, project promotion, and resourcing of appropriate initiatives. Given the benefits of student-led initiatives, institutions can be advocates for these activities within their courses, to ensure that all students have access to opportunities that interest them. We recommend that medical school faculty are more actively involved as mentors to student leaders and provide the connections with university alumni networks that will add impetus to international initiatives. Finally, we acknowledge the potential benefits of student-led initiatives which have not been rigorously assessed and support more research into the quality and impact of these activities. Included in this research, should be thorough assessments of the effects of international projects and student visits on host institutions.

\section{References}

[1] C. Bateman, T. Baker, E. Hoornenborg, and U. Ericsson, "Bringing global issues to medical teaching," The Lancet, vol. 358, no. 9292, pp. 1539-1542, 2001.
[2] M. K. Ali and K. M. Narayan, "The United States and global health: inseparable and synergistic? The institute of medicine's report on global health," Global Health Action, pp. 1-8, 2009.

[3] J. Frenk, L. Chen, Z. A. Bhutta et al., "Health professionals for a new century: ttransforming education to strengthen health systems in an interdependent world," The Lancet, vol. 376, no. 9756, pp. 1923-1958, 2010.

[4] M. K. Ali, J. M. Grund, and J. P. Koplan, "Case competitions to engage students in global health," The Lancet, vol. 377, no. 9776, pp. 1473-1474, 2011.

[5] General Medical Council, Tomorrow's Doctors: Recommendations on Undergraduate Medical Education, General Medical Council, London, UK, 2002.

[6] Accreditation Council for Graduate Medical Education, Core Competencies. Chicago, Ill, USA, ACGME, 2002, http://www .acgme.org/acwebsite/RRC_280/280_corecomp.asp.

[7] W. Pinar, Understanding Curriculum: An Introduction to the Study of Historical and Contemporary Curriculum Discourses, Peter Lang, New York, NY, USA, 1995.

[8] A. Bleakley, "Curriculum as conversation," Advances in Health Sciences Education, vol. 14, no. 3, pp. 297-301, 2009.

[9] World Federation for Medical Education, Basic Medical Education: WFME Global Standards for Quality Improvement, WFME, Copenhagen, Denmark, 2003.

[10] L. Hanson, S. Harms, and K. Plamondon, "Undergraduate international medical electives: some ethical and pedagogical considerations," Journal of Studies in International Education, vol. 15, no. 2, pp. 171-185, 2011.

[11] P. Drain, A. Primack, D. Hunt, W. Fawzi, K. Holmes, and P. Gardner, "Global health in medical education: a call for more training and opportunities," Academic Medicine, vol. 82, no. 3, pp. 226-230, 2007.

[12] R. F. Woollard, "Caring for a common future: medical schools' social accountability," Medical Education, vol. 40, no. 4, pp. 301-313, 2006.

[13] G. J. Fox, J. E. Thompson, V. C. Bourke, and G. Mononey, "Medical students, medical schools and international health," The Medical Journal of Australia, vol. 187, no. 9, pp. 536-539, 2007.

[14] S. Singh, "Global health in New Zealand: opportunities abound," New Zealand Medical Student Journal, no. 13, article 7, 2011.

[15] C. Deng, "Fiji village project: treating the patient, the water or the village?" New Zealand Medical Student Journal, vol. 10, pp. 20-21, 2009.

[16] J. Villafuerte-Galvez, W. H. Curioso, and J. J. Miranda, "The role of medical students in the fight to control neglected tropical diseases: a view from Peru," PLoS Neglected Tropical Diseases, vol. 2, no. 9, article e292, 2008.

[17] R. Duvivier, M. Mansouri, D. Iemmi, and S. Rukavina, "Migrants and the right to health: the students' perspective," The Lancet, vol. 375, no. 9712, p. 376, 2010.

[18] I. Sakon, "Nutrition and health-from a medical student organization," Japenese Medical Association Journal, vol. 51, no. 5, pp. 347-348, 2008.

[19] N. Arya, "Peace through health II: a framework for medical student education," Medicine, Conflict, and Survival, vol. 20, no. 3, pp. 258-262, 2004.

[20] R. Mularski, E. Young, D. Ornish, and C. Thomson, "Essentials of nutrition education in medical schools: a national consensus. American medical student association's nutrition curriculum project," Academic Medicine, vol. 71, no. 9, pp. 969-971, 1996. 
[21] J. Hilgers and P. De Roos, "European core curriculumthe students' perspective, Bristol, UK, 10 July 2006," Medical Teacher, vol. 29, no. 2-3, pp. 270-275, 2007.

[22] B. A. Palmer, A. Wong, and M. Singla, "Hearing the voice of medical students worldwide," PLoS Medicine, vol. 2, article e99, pp. 280-281, 2005.

[23] R. J. Duvivier, N. R. Watts, S. Rukavina, and C. Kaduru, "Doctors talk climate change-students take action," The Lancet, vol. 377, no. 9770, article 995, 2011.

[24] Subsidiary Body for Implementation of the UNFCCC, Report on essential needs for, potential gaps in, barriers to, and progress in the implementation of the amended New Delhi work programme, Cancun, Mexico, United Nations Framework Convention on Climate Change (UNFCCC), 2010.

[25] J. A. Crump and J. Sugarman, "Ethical considerations for short-term experiences by trainees in global health," Journal of the American Medical Association, vol. 300, no. 12, pp. 1456$1458,2008$.

[26] J. A. Crump, J. Sugarman, M. Barry et al., "Ethics and best practice guidelines for training experiences in global health," American Journal of Tropical Medicine and Hygiene, vol. 83, no. 6, pp. 1178-1182, 2010.

[27] A. Bleakley, J. Bligh, and J. Browne, "Global medical education-a post-colonial dilemma," in Medical Education for the Future: Identity, Power and Location, A. Bleakley, J. Bligh, and J. Browne, Eds., Springer, New York, NY, USA, 2011. 


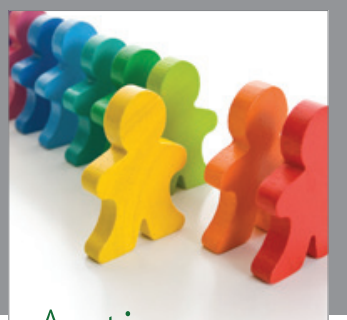

Autism

Research and Treatment
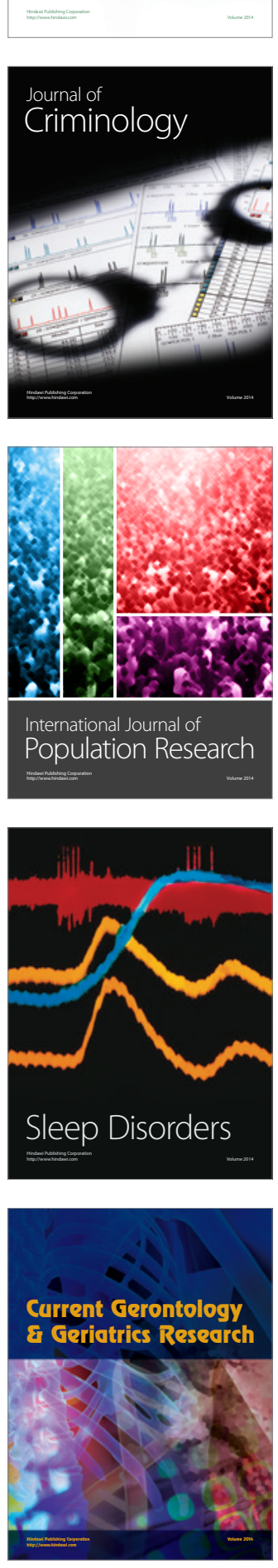
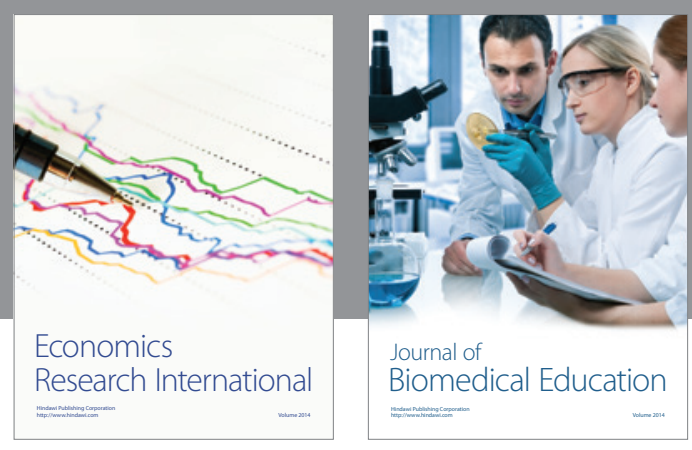

Journal of

Biomedical Education

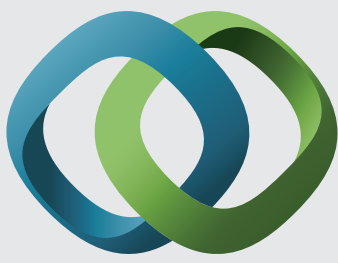

\section{Hindawi}

Submit your manuscripts at

http://www.hindawi.com
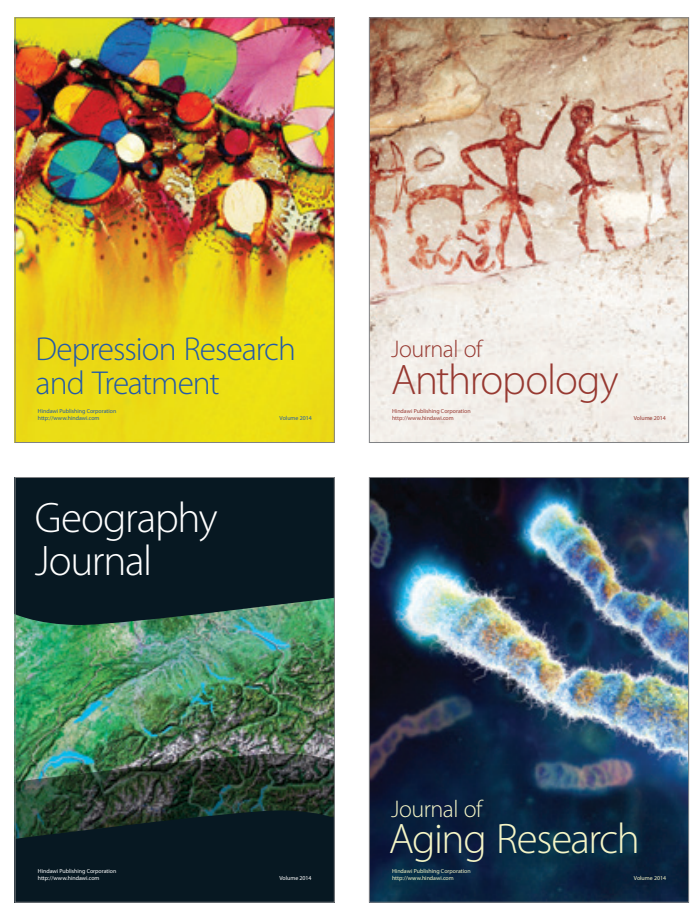

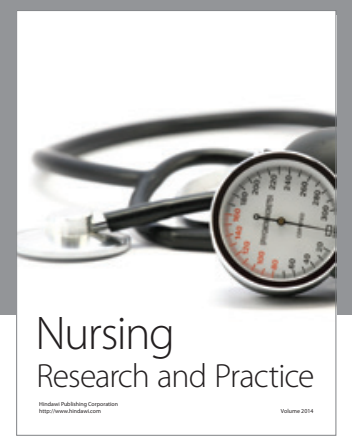

Nursing

Research and Practice

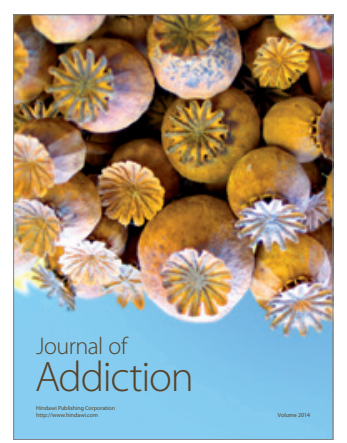

Child Development

Research

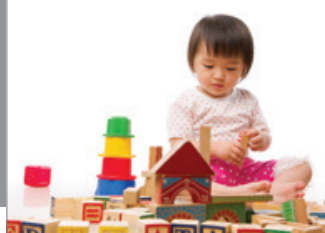

迥
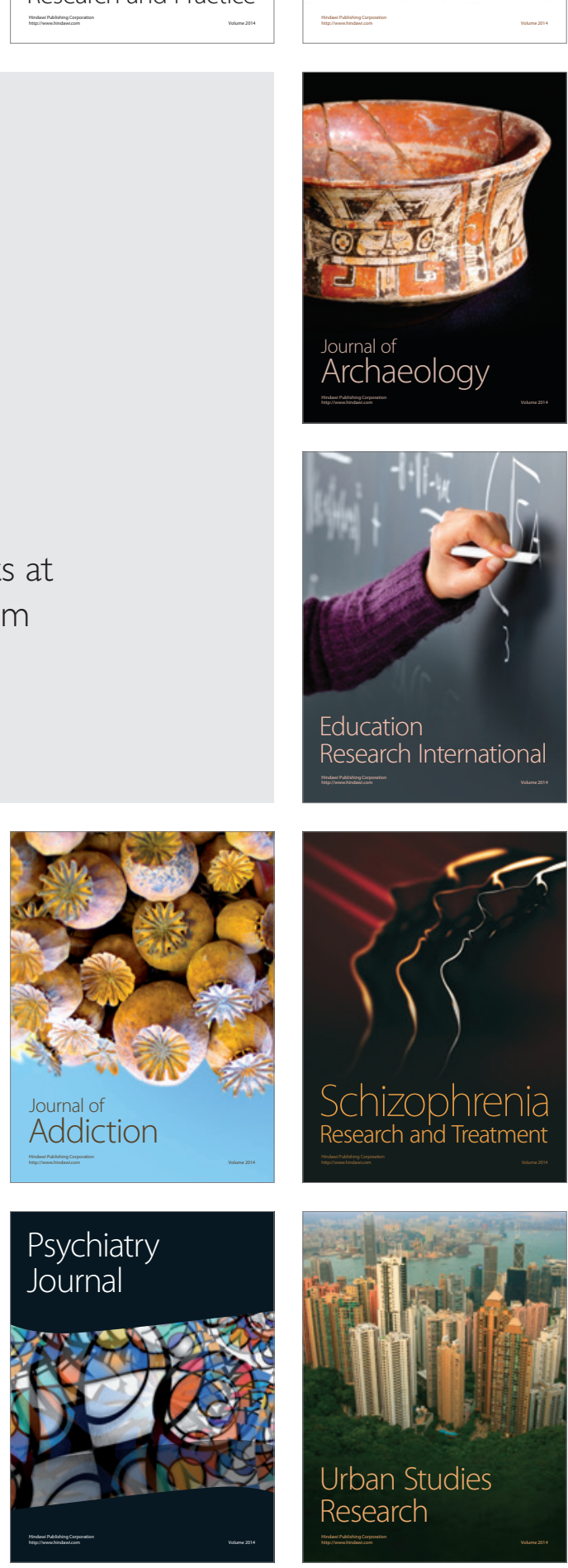